\title{
Preface
}

\section{Taming the Complexity: Using \\ Artificial Intelligence in a \\ Cross-Disciplinary Innovative \\ Platform to Redefine \\ Molecular Imaging and \\ Radiopharmaceutical Therapy}

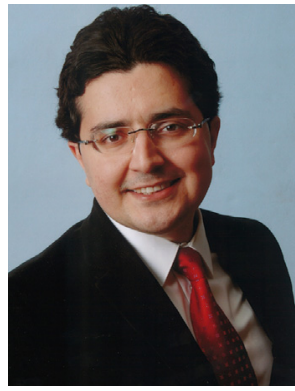

Babak Saboury, MD, $\mathrm{MPH}, \mathrm{DABR}, \mathrm{DABNM}$

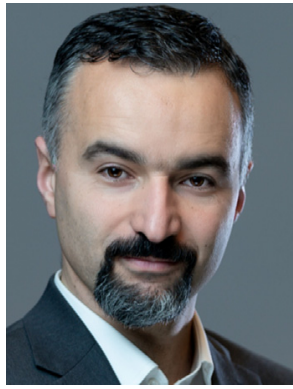

Arman Rahmim, PhD, DABSNM

Editors

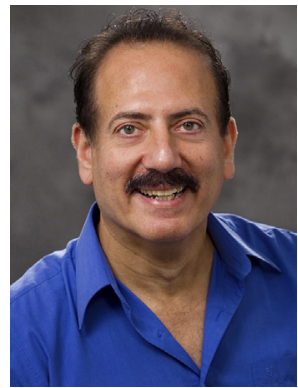

Eliot Siegel, MD, FSIIM, FACR
We are on the cusp of a major transformation in medicine. While the latter half of the twentieth century could be considered the age of computation and connectivity, the twenty-first century ushered in the era of big data accompanied by the rapidly evolving use of Artificial Intelligence (Al), dominantly in the form of deep learning, to "tame" the complexity associated with the ever-quickening velocity of information, including in molecular imaging and radiopharmaceutical therapy. Advanced $\mathrm{Al}$ algorithms have the potential to powerfully capture complex biological data and to be used to make improved predictions.

In the first issue of this collection, our colleagues addressed the use of Al to address complex technical challenges in nuclear medicine and PET imaging, such as improved image reconstruction and enhancement, as well as image segmentation and radiomics, radiopharmaceutical dosimetry, and treatment response evaluation.

The second issue considers practical considerations in the implementation of $\mathrm{Al}$ in clinical practice as well as the current state-of-the-art in specific clinical domains.

Saboury and colleagues discuss trustworthy Al in medical imaging, pointing out that Al systems are more opaque than human health care providers and are susceptible to being "fragile and unjust," which could result in breaches of trust in not only Al but also health care in general. They review fourteen core principles and requirements for trustworthy Al systems. In a thematically related article, they point out the critical importance of making $\mathrm{Al}$ algorithms aware of rare diseases, which occur in one in ten individuals, but which are rarely included in most image databases that provide training for Al algorithms. They suggest that $\mathrm{Al}$ algorithms should be made rare disease "aware" at each stage of their conceptualization and life cycle and should be trained on diverse and augmented data sets, including rare diseases.

To address an important but less explored aspect of $\mathrm{Al}$, Mezrich "demystifies" anticipated medicolegal challenges associated with Al for molecular imaging, suggesting that the biggest hurdles to Al in nuclear medicine may be more legal than technical and advocating for the establishment of guidelines and legislation that he believes are currently poorly delineated.

Critical appraisal of scientific evidence is an important ingredient of a trustworthy $\mathrm{Al}$ ecosystem. Steiner and colleagues advocate for 
an evidence-based approach to evaluation of $\mathrm{Al}$ in medical imaging using five basic questions, including the training sample, how a model is trained, reliability, validation, and usability.

Al for PET imaging has the potential to help to reinvent workflow, and as Beegle and colleagues suggest, put patients at the center of care. They focus on the application of Al for PET technologists, which they believe will improve communication with patients and allow technologists to replace current "mundane" tasks with more "challenging and satisfying tasks."

This second issue also explores the current state-of-the-art Al applications for PET for eight clinical domains, including Brain, Head and Neck, Lung, Heart, Vascular System, Bone/ Skeleton, Prostate, and Lymphoma.

Cross and colleagues describe the current state-of-the-art in the most widely written about area of Al in PET imaging, that is, applications in the brain. They organize articles about $\mathrm{Al}$ and PET into three tiers: workflow/image generation emphasizing quality and dose optimization; cognitive skills emphasizing decision making and progression of disease; and finally, "superhuman" algorithms that provide analysis not currently possible by human experts.

Gharavi and colleagues focus on prediction of response to treatment and tumor markers for head and neck cancers, including tumor and nodal segmentation and how to deal with the paucity of head and neck PET/computed tomographic (CT) data sets.

Zukotynski and colleagues review the use of $\mathrm{Al}$ in detection and characterization of lung nodules and predictive and prognostic biomarkers in patients with lung cancer, including staging, detection of recurrence, and outcome prediction.

Slomka and colleagues describe another area of very active research: cardiac PET/CT Al applications, including attenuation correction, dose reduction, and diagnostic and prognostic improvement.

Saboury and colleagues discuss bottlenecks to clinical adoption of PET for vascular diseases and predict that $\mathrm{Al}$-based segmentation will bring forth a revolution in more general applications of PET for quantification and phenotyping of these disorders.

Paravastu and colleagues focus on progress with Al applications in ${ }^{18} \mathrm{~F}-\mathrm{NaF}$ PET/CT in diagnosis, prognostication, and improvement of care for patients with bone diseases.

Prostate cancer imaging using relatively new targeted PET radiopharmaceuticals, such as PSMA, FACBC, and choline, is discussed by $\mathrm{Ma}$ and colleagues, who review the current medical literature and predict translation of current research into routine clinical practice using $\mathrm{Al}$.

Hasani and colleagues conducted an extensive scoping review of the current literature on quantification of disease burden in patients with lymphoma using $\mathrm{Al}$ and PET as well as treatment response and diagnosis and predictions of prognosis. They also review the literature on determination of change over time with an emphasis on going from just the spatial domain to the "spatiotemporal realm."

The second issue concludes with two forwardlooking articles. Ullah and Levin describe how Al will improve the design and performance of future PET scanners, from more accurate photon detection to more efficient data correction methods for quantitative imaging. Yousefirizi and colleagues review the concept of "Radiophenomics," a combination of radiomics and analysis of personalized structural and functional patterns of disease or drug response toward detection, classification, and outcome prediction.

We hope that despite the extraordinarily rapidly evolving advances in the application of $\mathrm{Al}$ in nuclear medicine, this review of the current state-of-the-art as applied to a variety of diseases and anatomic areas will provide a useful update and guide that will inspire the reader to continue to explore the evolution of $\mathrm{Al}$ in PET/CT. The application of machine learning and deep learning for PET/CT will undoubtedly result in major improvements in all aspects of clinical practice by advancing our ability to detect and diagnosis and treat disease more effectively and enhance patient safety and communication. Some have referred to Al as a "genie in a bottle," which speaks to its tremendous power and potential but should also serve as a cautionary 
metaphor to warn us of the potential dangers and challenges associated with its broad application in nuclear medicine without a well-informed understanding of its limitations and pitfalls.

Babak Saboury, MD, MPH, DABR, DABNM Department of Radiology and Imaging Sciences Clinical Center National Institutes of Health $(\mathrm{NIH})$ 9000 Rockville Pike Bethesda, MD 20892, USA

Department of Radiology Hospital of the University of Pennsylvania

Department of Computer Science and Electrical Engineering University of Maryland Baltimore County
Arman Rahmim, PhD, DABSNM Departments of Radiology and Physics University of British Columbia BC Cancer Research Institute 675 West 10th Avenue Office 6-112 Vancouver, BC V5Z 1L3, Canada

Eliot Siegel, MD, FSIIM, FACR Department of Radiology University of Maryland School of Medicine 655 West Baltimore Street Baltimore, MD 21201, USA

E-mail addresses: babak.saboury@nih.gov (B. Saboury) arman.rahmim@ubc.ca (A. Rahmim) esiegel@umaryland.edu (E. Siegel) 